\title{
Labyrinthe
}

37 | 2011 (2)

Des séries et des vies

\section{Des concepts et des faits}

La double contradiction des sciences sociales

\section{Bastien Bosa}

\section{(2) OpenEdition}

Journals

Édition électronique

URL : http://journals.openedition.org/labyrinthe/4202

DOI : $10.4000 /$ labyrinthe.4202

ISSN : 1950-6031

Éditeur

Hermann

\section{Édition imprimée}

Date de publication : 15 août 2011

Pagination : 121-147

ISBN : 9782705681470

Référence électronique

Bastien Bosa, « Des concepts et des faits », Labyrinthe [En ligne], 37 | 2011 (2), mis en ligne le 01 août 2013, consulté le 19 avril 2019. URL : http://journals.openedition.org/labyrinthe/4202 ; DOI : 10.4000/ labyrinthe.4202 


\title{
Des concepts et des faits La double contradiction des sciences sociales
}

\author{
Bastien Bosa*
}

\begin{abstract}
«À quoi sert l'histoire », demandait déjà un de ses jeunes fils à Marc Bloch, et chacun a lu la réponse. À « collectionner les faits », dit notre géographe ${ }^{1}$.
\end{abstract}

Il est clair que, s'il y a trois choses claires, elles sont quatre. Mais cellelà, qu'il y a trois choses claires, reste obscure ${ }^{2}$.

Je souhaiterais dans cet article explorer deux contradictions fondamentales pour définir l'espace logique dans lequel se déploient les sciences sociales. Celles-ci ont bien des difficultés, depuis leur émergence à la fin $\mathrm{du} \mathrm{XIX}$ " siècle, à définir leur statut, perdues dans une espèce " d'entre deux ", n'étant ni du côté des sciences de la nature, ni de celui des humanités classiques (ou alors appartenant aux deux simultanément). Paradoxalement, et comme l'avait bien souligné G. Friedmann (1960), les chercheurs en sciences sociales peuvent être perçus à la fois comme des « littéraires », des « gens pas sérieux, adonnés à de vaines et indémontrables spéculations » ou, au contraire, « comme des techniciens, pédants de la statistique et de l'enquête, étrangers aux problèmes essentiels de la culture, - en somme de dangereux représentants de la barbarie moderne ».

Si ces deux représentations contrastées me paraissent également trompeuses, elles ne sont pourtant pas sans fondement. Pour les comprendre, il faut, me semble-t-il, repartir de l'observation de J.-C. Passeron (1996 : 163) suivant laquelle le statut logique des propositions des chercheurs en sciences sociales se situe " dans l'entre-deux de l'observation singulière et du concept universel ». Cette idée implique de reconnaitre l'exis-

\footnotetext{
* Profesor Asociado, Universidad del Rosario, Bogota, Colombia.

1. R. Mandrou $1957: 620$.

2. Cité par J.-C. Passeron (1997 : 192).
} 
tence d'une double tension insoluble. D'un côté, les sciences sociales sont des sciences des " faits ", mais qui ne croient pas à l'existence de ces derniers (de peur de retomber dans des formes d'empirisme précritique). De l'autre, ce sont des sciences analytiques qui ne croient pas aux « concepts » au sens fort du terme (stigmatisant les tentations de l'abstraction et du formalisme scientiste). Si l'existence de cette double tension est largement reconnue, il n'y a pourtant pas d'accord sur son implication pour les pratiques quotidiennes de recherche. Il me semble possible, pour simplifier, de dégager quatre manières principales de faire face au problème. Une première position est occupée par ceux qui continuent à penser que les sciences sociales peuvent s'approcher d'une scientificité « véritable » : soit parce qu'ils considèrent que la reconstitution des « faits » constitue malgré tout l'horizon des chercheurs ${ }^{3}$, soit parce qu'ils pensent que les chercheurs ont vocation à proposer des « schémas conceptuels » formalisables rendant compte du fonctionnement du monde social $^{4}$. Une deuxième position consiste au contraire à disqualifier définitivement toute possibilité de discours scientifique sur le social: dans cette optique, il serait tout autant illusoire de prétendre décrire la " réalité » empirique que de vouloir proposer des concepts ou des théories pour la rendre intelligible. Au bout du compte, le chercheur ferait donc mieux d'assumer le caractère nécessairement littéraire et/ou imaginaire de son travail pour se livrer à l'interprétation libre des faits ${ }^{5}$. Une troisième position, qui est peut-être la plus fréquente statistiquement, consiste à décréter que le problème est un faux débat, ou pour le moins, qu'il serait contre-productif d'y consacrer trop de temps car il ne fait guère avancer les choses. Il vaut mieux continuer à travailler en « bricolant », quitte à devoir s'accommoder de certaines contradictions ${ }^{6}$. Enfin, une dernière

3. Cette tendance se présente le plus souvent comme une réaction contre les « excès » du relativisme et du postmodernisme. Certains historiens - certes peu légitimes au sein de la discipline, mais parfois très visibles auprès du grand public - continuent à penser, par exemple, que leur première mission est de rendre compte du passé tel qu'il s'est produit. Dans certains cas, cette référence à la « réalité des faits » se mêle à des considérations politiques. Cf. par exemple les history wars qui ont marqué les études aborigènes et animé le débat public en Australie tout au long des années 2000.

4. Dans le cas de l'anthropologie, on pensera à l'ambition d'Alain Testart (1991) ou de Dan Sperber (1996), qui prêchent pour une épistémologie commune avec les sciences de la nature.

5. C'est, semble-t-il, la conclusion qu'ont tirée certains des travaux d'Hayden White (1973) en histoire, ou de Clifford et Marcus (1986) en anthropologie. D'où le rapprochement d'une partie des chercheurs en sciences sociales avec les études culturelles ou littéraires.

6. On a ainsi critiqué l'inconséquence de Paul Veyne lorsqu'il laissait penser que deux propositions contradictoires ou inconciliables pouvaient toutes deux être justes : « Veyne est trop informé pour l'ignorer 
position revient à accepter ces tensions comme tensions, c'est-à-dire à reconnaître tout à la fois leur centralité et l'impossibilité de leur donner une solution définitive (puisque l'on ne peut pas choisir entre les deux positions de façon contraignante).

Les deux premières solutions peuvent apparaître comme les plus conséquentes. Elles ont en particulier le mérite de trancher les débats: si ces oppositions sont indépassables, il faut bien choisir. Pourtant, ceux qui manifestent pleinement leur confiance en les ambitions empiriques et/ou théoriques des sciences sociales, comme ceux qui les mettent en cause radicalement, n'ont souvent d'autre choix que d'accepter des vérités simples et définitives, quand tout indique qu'il est toujours possible de « tordre le bâton dans l'autre sens ». Réciproquement, ceux qui pensent que l'alternative est un faux problème ont trop souvent tendance à se contenter de commodités de langage qui résoudraient magiquement toutes les difficultés. Pourtant, ces dernières restent le plus souvent intactes.

Enfin, la dernière attitude ne prétend certes pas résoudre cette double tension qui est au cour du travail des chercheurs en sciences sociales, mais en se proposant de l'expliciter, elle a l'avantage de permettre d'avancer: les apories ou les difficultés mal résolues ne sont plus considérées dans ce cas comme des dilemmes stérilisants mais comme des tensions tout à la fois stimulantes et indépassables. Évidemment, cette dernière position ne peut jamais être entièrement satisfaisante, puisqu'elle consiste à reconnaitre que l'on est seulement capable d'identifier une série de difficultés, et non de les résoudre. C'est pourtant celle que nous retiendrons dans cet article, dont l'objectif, on l'aura compris, ne sera pas de proposer des solutions définitives, mais de clarifier la nature du débat.

\section{I- Des sciences des faits...}

Une première caractéristique centrale dans le travail des chercheurs en sciences sociales est liée à ce que l'on pourrait appeler leur « empirisme irréductible » (cf. Schwartz: 1993). C'est-à-dire que toute étude sociale doit

mais il mélange à plaisir les affirmations contradictoires, comme si de leur somme algébrique naissait un tableau équilibré. Quand un choix se présente, Veyne est simultanément pour les deux termes de l'alternative et pour aucun d'entre eux. Avec les concepts, il se comporte comme un pompier pyromane qui s'ingénie à provoquer des explosions pour aussitôt les éteindre. » (Cf. Schnapp et al. 1978). 
s'éprouver au contact du monde, à travers la collation, le déploiement et l'exploitation de matériaux de diverses natures. De manière traditionnelle, cette épreuve empirique a permis aux chercheurs en sciences sociales de se distinguer des essayistes ou des journalistes, qui, en particulier pour des questions de temps, peuvent se contenter d'une connaissance de seconde ou de troisième main des univers dont ils parlent ${ }^{7}$. Le chercheur ne s'autorise, lui, à parler d'un univers social que lorsqu'il en a acquis une grande familiarité, au terme d'une longue patience. Cette prétention à réunir, observer ou étudier patiemment des « faits » peut prendre des modalités très diverses et renvoyer à de multiples techniques d'enquête. Trois exemples possibles:

- L'ethnographie consiste à étudier longuement, minutieusement, et de manière directe et personnelle, un univers social. Le chercheur produit lui-même ses propres matériaux dans un journal de terrain élaboré à partir de son expérience et des relations qu'il a nouées avec ses enquêtés. Et ce sont ces notes de terrain qui, en grande partie, donne une légitimité aux résultats présentés, permettant à l'enquêteur de s'affirmer comme spécialiste de tel ou tel groupe.

- La recherche sur archive, c'est-à-dire le dépouillement de sources de première main, manuscrites ou imprimées, joue un rôle similaire pour les chercheurs qui s'intéressent au passé. S'il convient, nous y reviendrons, de ne pas confondre les traces sur lesquelles travaillent le chercheur et les faits eux-mêmes, évanouis, il est central de reconnaître que les documents constituent pour les chercheurs une contrainte essentielle, qui fixent des limites à leur discours. « On fait de l'Histoire en fréquentant les archives », écrivait L. Febvre (1953: 514), les présentant comme une étape préliminaire indispensable.

- La mesure des faits et la constitution de « corpus », enfin, constituent une autre épreuve empirique. L'enquête statistique et les méthodes de quantification fournissent aux chercheurs des données fondamentales, qui leur permettent de rendre compte de liens d'interdépendance entre des faits d'ordre divers ${ }^{8}$.

7. Encore qu'évidemment, il peut être difficile de situer précisément la frontière entre les genres essayiste et scientifique. «Qui veut noyer son chien, en sociologie, dit qu'il est essayiste », ironisait G. Lenclud (1991). Il arrive pourtant, continuait-il, « qu'on trouve plus de rigueur [...] chez certains essayistes que dans maints ouvrages de sciences sociales ».

8. On identifie habituellement ces trois techniques à chacune des disciplines des sciences sociales: l'ethnographie aux anthropologues, les archives aux historiens et les statistiques aux sociologues. 
Cette visée empirique des sciences sociales a également permis de les distinguer des analyses herméneutiques ou philosophiques qui peuvent, elles, restreindre leurs ambitions (mais c'est déjà beaucoup!) à une réflexion sur des concepts; et qui n'ont pas l'obligation, dans leur travaux, de faire systématiquement référence à la réalité sociale 9 . Pour les sciences sociales, au contraire, l'observation des " faits concrets » est une condition de toute recherche. D'une certaine façon, la réalité observable apparaît à la fois comme la visée des connaissances qu'elles génèrent (puisqu'il s'agit de rendre raison d'un monde donné) et comme leur sanction (puisque l'adéquation au réel permet de discriminer les « bonnes » et les « mauvaises » recherches). C'est ce qui explique que les sciences sociales se nourrissent d'exemples et de cas particuliers, stigmatisant, d'une part, ce qu'elles appellent parfois la « théorie théorisante » et, d'autre part, la prétention à établir ce qui est vrai toujours et partout ${ }^{10}$. Reprenant la phrase célèbre de Mauss, « ce qui compte, c'est le Mélanésien de telle ou telle île... », Lévi-Strauss (1960) défendait fermement ce credo empiriste: "Contre le théoricien, l'observateur doit toujours avoir le dernier mot; et, contre l'observateur, l'indigène ».

Dans tous les cas, l'observation et la description des faits sont, et demeurent, un élément indispensable, qui ne doit pas disparaître dans l'écriture finale: le lecteur doit pouvoir discuter les propositions avancées grâce à une confrontation avec des sources. Même si elle alourdit le récit et en rend la lecture moins fluide, cette référence permanente aux " matériaux » permet de ne pas isoler les résultats des processus concrets qui ont permis de les élaborer. Cette dernière idée est centrale pour l'ensemble des chercheurs, quels que soient les matériaux sur lesquels ils

\footnotetext{
Cette répartition est pourtant sans fondement puisque chaque technique a vocation à être utilisée par l'ensemble des chercheurs.

9. Cette présentation est évidemment une simplification. Si une partie des philosophes assument très clairement leur détachement des conditions concrètes du monde social, d'autres revendiquent au contraire un contact direct avec le monde réel. Pourtant, pour eux aussi, la question du rapport aux données empiriques fait problème. Le contentieux qui opposait Michel Foucault à certains historiens tournait par exemple en grande partie autour de cette question. Alain Cottereau faisait ainsi remarquer que Foucault avait seulement travaillé sur trois règlements d'atelier dans Surveiller et punir, quand lui-même en avait analysé plus de 700. Si le nombre lui-même importe peu, il est évident que cette différence d'échelle n'est pas sans conséquence sur le travail des uns et des autres. Il n'en reste pas moins que l'on ne peut qu'admettre qu'il n'existe pas de « seuil » fixe à partir duquel la dimension empirique d'un travail de recherche serait attestée.

10. L'une des caractéristiques principales des faits sociaux est en effet qu'il « se passent toujours à un moment du temps et en un endroit de l'espace » (cf. Simiand: 1903).
} 
travaillent. Malinowski insistait ainsi dès 1922 sur l'importance, pour l'ethnographe, d'abolir la distance entre le «matériau brut de l'observation » et « l'exposé ultime et apodictique des résultats » (de façon à ne pas « livrer (des) conclusions toutes faites, sans rien nous dévoiler de leur genèse »). De façon très similaire, Marc Bloch (1952:30) soulignait la nécessité, dans toute recherche, "d'insérer aux points tournants du développement, une suite de paragraphes qui s'intitulerait à peu près: "Comment puis-je savoir ce que je vais dire?" ».

\section{...qui ne croient pas aux faits}

La réalité [...] restera à jamais ce qu'elle a été (mais qui sait ce qu'elle a été?) ${ }^{11}$

L'historien doit procéder tout fait comme le poète. Quand il a assimilé la matière il doit la recréer de manière entièrement nouvelle partir de lui-même ${ }^{12}$.

II est une chose que jamais on ne voit ni n'entend: ce sont les faits historiques. Les faits historiques, on les reconstitue ${ }^{13}$.

Si cette dimension empirique des travaux en sciences sociales est fondamentale, elle n'est pas sans poser problème. En particulier, parce qu'avec elle apparaît la question de la définition de ce qu'est pour le chercheur la "réalité sociale », de ce que sont les «faits ». Or, pour progresser, les sciences sociales ont dû renoncer à la croyance en une transparence ou une possibilité de lecture directe des « situations réelles du monde réel ». Plus précisément, elles ont dû apprendre à distinguer clairement la "réalité », par essence inaccessible, et l'objet d'enquête, toujours construit, déformé, recomposé et recontextualisé ${ }^{14}$. Le chercheur n'a donc pas vocation à restituer les « faits » en tant que tels, mais à se les approprier; et le savoir qu'il produit ne provient jamais directement

11. Raymond Aron (1971 : 135).

12. Dans Hartog François. « G. de Humbolt, la tâche de l'historien », Annales. ESC, 1986, n 1, p. 94-95.

13. Georges Smets, 1926.

14. Cf. «Présentation ». Annales, 1997. Il n'est d'ailleurs pas très difficile de percevoir le décalage entre le monde « réel » et les récits qu'en font les sciences sociales. 
de la réalité, présente ou passée, mais d'un travail qui lui est propre. Il s'agit donc de reconnaître que le rêve de pouvoir restituer les « faits " grâce à une description exhaustive et méticuleuse de ce qui serait arrivé dans tout son détail et sa complexité est une illusion.

Cette idée que la « réalité » serait par essence inaccessible peut cependant prêter à confusion. Il faudrait par exemple être de bien mauvaise foi pour nier que l'on peut corriger Quentin Tarantino " au nom des faits », lorsqu'il montre dans l'un de ses films que Hitler s'est fait assassiner à Paris dans un cinéma en 1944. Ou Robert Guédiguian quand il met en scène la « rafle du Vel d'hiv » en $1943^{15}$. De même, il est évident que de nombreuses querelles entre chercheurs en sciences sociales ont porté sur des « erreurs factuelles »: le nombre de morts lors d'une guerre ou d'un massacre, la présence d'une personne lors d'une situation donnée, l'appartenance d'une autre à un groupe, la date ou le lieu d'un événement, le titre ou l'auteur d'un écrit, le contenu d'une norme ou d'un texte. Il n'est certes pas toujours possible de trancher définitivement ces controverses, mais la difficulté tient essentiellement aux lacunes de la documentation (c'est à dire à un problème technique plus qu'interprétatif). Ces énoncés ont donc en commun de renvoyer à un type de régime de vérité particulier : celui d'une relative « vérificabilité » / « véridicité ».

Or, une première erreur de ce que nous pourrions appeler «l'empirisme naïf » consiste à considérer que le travail des sciences sociales revient essentiellement à trancher ce genre de polémiques ou que le travail empirique se résume à l'établissement de «f faits vérifiables ». Pour ne pas nous tromper de débat, il nous semble nécessaire de commencer par différencier, de façon certes simplificatrice, les types d'énoncés que peuvent produire les chercheurs en sciences sociales ${ }^{16}$. D'un côté, ceux qui relèvent du domaine factuel (les dates, les lieux, les contenus, les comptages ${ }^{17}$, etc.) et qui entrent dans une logique du vrai et du faux au

15. Inglorious Basterds, Universal Pictures, 2009 et L'Armée du crime, StudioCanal, 2009. Bien entendu, ces deux « erreurs factuelles » sont conscientes et assumées par les deux réalisateurs. Ce n'est cependant pas toujours le cas.

16. À la différence des considérations épistémologiques qui entendent souligner la spécificité des énoncés des sciences historiques par rapport à ceux des sciences de la nature, il nous semble important de commencer par montrer qu'il existe, à l'intérieur même des sciences sociales, des énoncés renvoyant à des logiques diverses. Cf. la critique adressée par J.-P. Olivier de Sardan à J.-C. Passeron (1993).

17. Tous les comptages ne relèvent pourtant pas au même titre du «vérifiable »: compter les morts, par exemple, ce n'est pas pareil que compter les chômeurs ou les membres d'un groupe racial. À la différence du chômage ou des phénomènes de racialisation, le phénomène de la vie de la mort est en 
sens classique (et auxquels peut donc s'appliquer la notion de falsifiabilité). D'un autre côté, ceux qui relèvent d'une interprétation et qui ne peuvent prétendre qu'à un statut de « plausibilité » / « véracité » : établir ce que pensent les gens, individuellement ou collectivement, au-delà de ce qu'ils font ou de ce qu'ils disent (leurs états mentaux plus ou moins conscients), chercher les raisons ou même les causes de ces pensées, $s^{\prime}$ interroger sur les ressorts ou sur le sens de leurs actions et interactions ${ }^{18}$, etc. Pour ce type d'énoncés, il semble impossible d'appliquer de façon stricte des critères de vérité et de fausseté. Dans le meilleur des cas, ils seront vraisemblables ou convaincants.

Dire que les faits sont « construits », ce n'est donc pas affirmer qu'il est impossible de s'accorder sur le fait que tel événement s'est déroulé à telle date, ou que telle ou telle personne y était présente ${ }^{19}$. Ce n'est pas non plus nier la possibilité, pour l'enquêteur du contemporain, d'observer et de décrire, ou, pour le statisticien, de produire des données chiffrées sur le monde social. C'est par contre affirmer que les sciences sociales ne peuvent se limiter à l'établissement ou à l'accumulation de « petits faits vrais » (au fameux « art de vérifier les dates » ou à celui de « réciter le réel »). Si l'on peut très légitimement critiquer un auteur pour les erreurs factuelles dont il s'est rendu coupable (par exemple, ses failles dans le respect de la chronologie ou la pauvreté de sa base documentaire ${ }^{20}$ ), on peut tout aussi bien lui reprocher de s'en tenir à l'accumulation d'une série de « preuves » factuelles. C'est en effet une illusion de penser que la somme d'un ensemble d'informations, dont le seul point commun est que l'on peut en vérifier l'exactitude, est suffisante pour expliquer ou comprendre quelque chose du monde social. Une partie essentielle du travail des chercheurs commence précisément quand ils s'aventurent, au-delà de l'érudition ou de ce que Marc Bloch appelait les «fausses précisions », dans un travail de construction ou d'interprétation. Cette

effet indépendant des hommes et de leurs institutions. Avant de compter, il est nécessaire de se mettre d'accord sur la définition, toujours socialement et historiquement située, de ce qu'il faut compter.

18. Par exemple, le simple fait de qualifier des actions ou interactions comme « amicales » ou comme « violentes » (ou contraire comme « faussement » amicales ou « faussement » violentes) nécessite de la part du chercheur une interprétation (cf. Naepels, 2006).

19. « Je m'en tiens, quant à moi, aux faits. Henri IV a été assassiné le 14 mai 1610, rue de la Ferronnerie. Ce n'est pas une invention de l'esprit, ni une création de l'histoire », faisait ainsi dire L. Febvre (1956) à un contradicteur imaginaire.

20. On pourrait d'ailleurs regretter le faible nombre des mises en cause proprement « empiriques » (et non « analytiques ») dans les comptes rendus critiques, notamment dans le cas des travaux ethnographiques. 
situation est en partie liée à l'une des grandes spécificités des sciences sociales: les personnes que nous étudions savent (au moins en partie) ce qu'elles font et elles donnent du sens à leurs actions. Elles ont des motifs et des raisons qui font sens au sein de l'univers social auquel elles appartiennent ${ }^{21}$. Or, nous ne pouvons jamais faire abstraction de ces interprétations pour élaborer les nôtres et il n'est pas possible de parler de phénomènes humains ou sociaux en laissant de côté la question du sens ou de la signification ${ }^{22}$. Plus que de vouloir établir « la » véritable interprétation sur le fonctionnement du monde social, le chercheur doit donc prendre acte de la pluralité des interprétations et des regards, de façon à proposer ce que l'on pourrait appeler, avec Bourdieu (1993), « un point de vue sur des points de vue ${ }^{23}$ ».

Une deuxième critique de l'empirisme naïf passe par la reconnaissance de l'impossibilité d'être exhaustif. Le chercheur doit commencer par admettre que le monde social constitue une réalité ouverte qu'il ne pourra jamais saisir ou décrire définitivement. Dans le meilleur des cas, il s'agira d'en lever une carte aussi précise que possible, mais reposant nécessairement sur un nombre limité de faits tirés de la vie d'individus spécifiques. Or, l'idée suivant laquelle on ne peut jamais avoir qu'une vue partielle du monde social a mis longtemps à s'imposer, et ceci quelle que soit la méthodologie mise en œuvre. Tout d'abord, de nombreux anthropologues avaient l'illusion de pouvoir saisir et comprendre les cultures qu'ils étudiaient dans leur totalité, car celles-ci étaient considérées comme relativement « simples » (et donc à la portée « d'un seul esprit $\left.^{24} »\right)$. Et cette croyance était renforcée, au niveau collectif, par la

21. Cette caractéristique est probablement celle qui justifie avec le plus de force l'existence d'un espace épistémologique propre aux sciences sociales (peut-être plus encore que l'historicité singulière de leurs objets sur laquelle insiste principalement J.-C. Passeron). Une partie des économistes ont résolu ce problème des motifs en se basant sur l'idée de rationalité des acteurs (laquelle a l'avantage indéniable de permettre l'élaboration de modèles de calcul). Cette solution n'est pas entièrement satisfaisante, cependant, car l'on peut toujours trouver de bonnes raisons pour qu'une personne ne soit pas rationnelle au sens strict.

22. Cette idée peut être résumée de la façon suivante: «L'expérience des significations fait partie de la signification totale de l'expérience » (Bourdieu, Boltanski, Castel et Chamboredon, 1965).

23. Il subsiste une ambiguitté dans le texte de Bourdieu. Une interprétation forte laisse penser que le sociologue, au nom de la science, pourrait prétendre atteindre le « point de vue de tous les points de vue ». Une interprétation souple insiste au contraire sur le fait que cette ambition, non seulement, ne sera jamais définitivement acquise, mais qu'elle est pose problème au sens où rendre compte de la pluralité des points de vue, c'est ne pas vouloir les dissoudre ou les réduire à un seul (celui du chercheur). 24. Cf. Evans-Pritchard (1950). 
démarche d'inventaire et de collecte systématique qui a longtemps caractérisé la discipline. Il s'agissait de multiplier les monographies de façon à permettre, à terme, d'établir ce que Marcel Griaule (1957 :5) appelait les « archives totales de l'humanité ». Pourtant, on le sait bien, quelle que soit « l'épaisseur » d'une description ethnographique, elle ne pourra jamais épuiser la réalité. L'idée d'exhaustivité a également joué un rôle central chez les historiens. Là aussi, cette importance reposait pourtant sur une confusion: on assimilait la possibilité d'étudier l'ensemble des documents ayant survécu sur un thème particulier (ce que l'on pourrait appeler une « exhaustivité documentaire ») et celle de rendre compte de l'ensemble du monde social dont ces documents sont issus («l'exhaustivité analytique $»)^{25}$. Or, bien évidemment, les deux choses sont très différentes : l'accès à l'ensemble des documents connus sur un sujet donné (qui, en soi, n'a rien d'évident, étant donnée l'élasticité presque infinie des sources possibles) est loin de garantir une connaissance complète du monde étudié ${ }^{26}$. Enfin, les analyses statistiques ou sérielles ont, elles aussi, eu tendance, à certaines époques, à poursuivre le vain rêve de l'exhaustivité. De façon plus ou moins implicite, le développement de banques de données chaque fois plus vastes était pensé comme le moyen, à terme, d'une saisie complète du « réel ». Or, cette démarche est, elle aussi, apparue comme illusoire puisque l'accumulation des informations se faisait bien souvent aux dépends du souci même de l'interprétation. Loin de permettre une reproduction de la totalité objective du fait, cette démarche conduisait essentiellement à la production d'artefacts.

La troisième idée centrale pour une critique de l'empirisme est liée au fait que toute perception du monde social est non seulement partielle mais partiale. Il s'agit simplement de reconnaître ici que tout processus de vision implique des points aveugles. Et ceci, autant au moment de la collecte des matériaux qu'à celui de leur analyse. Tout d'abord, que l'on travaille par enquête directe, sur archives ou à partir de données chiffrées, l'élaboration des corpus n'est jamais un acte neutre. Dans le cas de l'ethnographie, il est évident que le chercheur joue un rôle fondamental dans

25. Il me semble que c'est la confusion que faisait Bernard Cohn (1962), lorsqu'il présupposait qu'à la différence de l'anthropologue, un historien pouvait « réaliser une étude exhaustive », dans la mesure où « seulement une partie de la documentation avait survécu».

26. En ce sens, le fait de parler des «lacunes de la documentation» ou du « vide entre les sources » est trompeur, car cela laisse penser qu'il serait possible de se mettre en quête de l'exhaustivité pour proposer une version « définitive » des faits. 
la production des matériaux puisque ceux-ci sont le résultat d'interactions entre lui-même (comme personne sociale) et le milieu enquêté. En ce sens, la dynamique d'enquête est toujours étroitement dépendante des choix et de l'attitude du chercheur. Mais même dans le cas d'enquête sur archives ou statistiques, la constitution des corpus est inséparable des préférences, conscientes ou non, du chercheur, ainsi que d'un ensemble de présupposés qu'il est indispensable d'expliciter ${ }^{27}$. Indépendamment de sa méthode d'enquête, ce que le chercheur voit et ce qu'il ignore, ce qui l'intéresse ou au contraire l'ennuie, dépend inévitablement de sa problématique, de ses curiosités, de ses convictions ou de ses expériences.

Et cette opération de sélection se répète au moment de l'analyse: les matériaux (encore une fois, leur nature importe peu) ne font pas sens par eux-mêmes et ils ne fournissent pas non plus spontanément les questions au chercheur. C'est à ce dernier de déterminer les problèmes essentiels, de mettre en avant certains aspects et d'en éliminer d'autres, d'organiser et de faire des choix. Rien n'est donc plus trompeur que l'idée selon laquelle il existerait un matériel brut, se suffisant à lui-même et qu'il suffirait de transcrire.

$\mathrm{Au}$ final, une recherche en sciences sociales ne peut donc jamais atteindre « le sens ultime » des phénomènes. C'est, nous y reviendrons, une construction partielle et révisable, s'appuyant sur des méthodes ou des modèles d'intelligibilité différents. En ce sens, si l'érudition et les matériaux empiriques sont indispensables aux chercheurs en sciences sociales, ils ne doivent pas être fétichisés et ils ne constituent pas une fin en soi. Ils n'ont pas de valeur sans un travail de construction du chercheur, et ces constructions sont toujours provisoires.

\footnotetext{
27. Le chercheur qui travaille sur archives doit par exemple s'interroger de manière réflexive sur toute la « trajectoire » d'un document auquel il a recours pour ses analyses : les conditions de sa production, celles de sa « mises en archives » et de sa conservation (pour lesquelles le chercheur n'a généralement pas eu d'implication personnelle), mais aussi celles de sa « mise en corpus » et de son analyse (pour lesquelles le travail du chercheur joue un rôle fondamental).
} 


\section{Un classique des sciences sociales}

L'idée suivant laquelle les sciences sociales ont à construire leur objet pour aller au-delà d'une saisie naïve du réel n'est pas une invention postmoderne, mais bien un grand classique de l'histoire des sciences sociales. Saussure insistait ainsi sur le fait que « le point de vue crée l'objet » et toute la tradition sociologique, notamment durkheimienne, s'est construite sur l'idée d'une conquête du fait sociologique (en particulier à travers l'idée de " construction de l'objet »). Et cette idée a été reprise plus tard par Marc Bloch et Lucien Febvre, dans leur valorisation d'une « histoire-problème » plutôt que d'une « histoire-récit ». Contre l'empirisme descriptif qui avait longtemps dominé les recherches historiques, ils insistaient sur l'importance, pour orienter l'enquête, de poser des problèmes et formuler des hypothèses. On se souviendra ainsi de leurs fortes récriminations contre les « tenants de l'érudition pour l'érudition ", contre les « chevaliers du fait pour le fait ${ }^{28} 》$ :

En ce temps-là, les historiens vivaient dans un respect puéril et dévotieux du « fait». Ils avaient la conviction, naïve et touchante, que le savant était un homme qui, mettant l'œil à son microscope, appréhendait aussitôt une brassée de faits ${ }^{29}$.

\section{II- Des sciences analytiques...}

Un historien ne fait pas parler les Romains, il parle à leur place. ${ }^{30}$

Si les costumes et les décors appartiennent aux Nambikwara, le texte et la mise en scène sont de Claude Lévi-Strauss. ${ }^{31}$

La deuxième caractéristique centrale des sciences sociales est leur ambition " analytique ». Précisément parce qu'ils entendent dépasser l'empirisme descriptif, les chercheurs se doivent de mobiliser dans leur

28. Lucien Febvre (1953: 68).

29. Lucien Febvre (1943: 9).

30. François Hartog $(1978: 328)$, citant Paul Veyne.

31. Bernard Lahire (1996: 73) 
recherche des formes de théorisation, des armatures conceptuelles, qui, seules, permettent d'élaborer des hypothèses et de mettre en forme les données. Le recours à la théorie apparaît donc comme la conséquence logique de l'idée suivant laquelle le chercheur ne peut jamais atteindre complètement la singularité des événements humains.

Tout d'abord, chaque fois qu'un chercheur prend pour objet un événement absolument singulier (un assassinat, une grève, etc.), il n'a pas d'autre choix (ne serait-ce que pour le « décrire ») que de le rapprocher, de façon plus ou moins explicite, d'autres phénomènes qui, bien que dotés eux aussi de propriétés irréductibles, présentent des caractéristiques générales analogues (la classe des assassinats politiques, des grèves insurrectionnelles, etc. $)^{32}$. Mais, il semble possible d'affirmer, de manière plus générale, qu'il est impossible de « ne pas théoriser » (c'est-à-dire de décrire sans concepts ou sans interprétation). En effet, qu'ils en aient conscience ou non, les chercheurs, chaque fois qu'ils énoncent un fait, doivent mettre en œuvre une théorie implicite de ce qu'ils décrivent ${ }^{33}$. Comme le soulignait bien Paul Veyne (1969), « qui n'a pas de théorie a ses préjugés » (ou, rajouterait Bourdieu, ceux de ses enquêtés). Ainsi, un chercheur qui voudrait rendre compte des ressorts de l'action sans constructions conceptuelles a de grandes chances de mettre en œuvre une théorie implicite pour laquelle les besoins psychologiques des individus constituent la raison suffisante de leurs pensées et actions. Loin d'être neutre, ce présupposé implique de renoncer à prendre en compte l'inscription sociale, historique ou culturelle des pratiques, pour placer au centre de l'analyse les actions intentionnelles des personnes. Jean-Pierre Peter (1964) illustrait ce point dans un compte rendu critique d'un livre d'Hugh Thomas consacré à la guerre d'Espagne. En apparence, l'auteur y décrivait de manière purement factuelle les premières conséquences de la rébellion militaire à Valence en juillet 1936, où le général Carrasco avait échoué et s'était enfui : « Ses partisans restèrent bloqués dans les casernes; onze églises furent incendiées et l'Archevêché détruit ». Or, comme le montrait J.-P. Peter, le simple fait de livrer ces deux « informations » côte à côte, impliquait déjà une grille de lecture. Si l'auteur n'avait certes pas établi entre elles un champ de corrélations explicites (ce qui lui permettait de

32. R. Aron (1971 : 1348) expliquait ainsi qu'« il n’y a[vait] pas eu deux empires romains, mais il y a[vait] eu maints empires ».

33. En ce sens, décrire, ce n'est pas seulement observer mais également déchiffrer. 
sauvegarder les apparences de la neutralité ou de la non-intervention), la juxtaposition des deux images invitait en fait à une lecture très orientée de la réalité, insistant sur l'instinct sacrilège de foules déchaînées ou sur l'existence d'un pur désordre. On contrastera cette absence de réflexion sur les concepts avec les travaux de l'anthropologue Philippe Bourgois (2002), qui a lui proposé de distinguer quatre types de violence: quotidienne, symbolique, structurale et politique. Ce travail de conceptualisation est précisément un moyen pour Bourgois de prendre ses distances avec la notion de « violence » telle qu'elle est utilisée dans le sens commun. Il lui permet à la fois de rapprocher des éléments habituellement séparés (par exemple les dimensions « physiques » et « morales » de la violence) ou, au contraire, de séparer des dimensions généralement réunies (par exemple, la violence policière et celle des gangs). Il lui permet également de prendre en compte des éléments occultés par le sens commun (par exemple la question du « consentement» à travers le concept de « violence symbolique ») ou d'établir des relations non perçues entre des ordres de faits (par exemple, le lien entre des formes « invisibles» de violence - disons l'expérience du chômage telle que pensée à travers le concept de « violence structurale »- et des formes « visibles », comme les expressions ordinaires d'agressions interpersonnelles).

Il est donc illusoire de penser qu'il serait possible pour le chercheur de s'effacer, de s'abstenir de toute intervention, de façon à ne pas dénaturer l'intégrité ou le sens des matériaux. Si, comme nous l'avons vu, les matériaux empiriques ne peuvent parler seuls, il faut bien, pour les rendre intelligibles, que le chercheur les soumette à une activité analytique et organisatrice: comparer, interroger, formuler des hypothèses, voire des schémas explicatifs ou des «modèles ». Ces derniers sont autant de points d'appui qui permettent d'effectuer une représentation, évidemment plus ou moins simplifiée, des phénomènes réels. La théorie s'apparente donc à une forme de réduction (qu'il ne faut pas confondre avec un réductionnisme), qui permet d'effectuer une « transposition » du réel. Au final, pour être considérées comme valides, les propositions ne doivent donc pas seulement subir une épreuve empirique: elles doivent faire sens à l'intérieur du système de propositions dans lequel elles s'insèrent. Elles doivent faire l'objet de ce que l'on appelle généralement 
une " problématisation », qui implique de s'intéresser aux faits, non plus comme faits, mais « comme outils et comme problèmes $»^{34}$.

\section{...qui ne croient pas aux concepts}

Les événements n'ont pas d'unité rationnelle; on ne peut, comme le bon cuisinier du Phèdre, les découper selon leurs articulations véritables, car ils n'en ont pas. ${ }^{35}$

Pour autant, cette reconnaissance du rôle central de la théorie dans les recherches en sciences sociales ne résout pas toutes les difficultés. Là aussi, si l'idée que la recherche en sciences sociales suppose la conceptualisation est aujourd'hui largement acceptée, il semble pourtant qu'il n'y ait pas d'accord sur le sens ou les implications de cette affirmation. Le premier problème est donc lié à la définition de l'opération de théorisation, dont on peut distinguer une conception « faible » et une conception « forte ». Pour certains, insister sur l'importance des procédures analytiques revient simplement à affirmer que le chercheur doit faire un travail de construction et de mise en ordre de ses matériaux. Il doit trouver des cadres et des catégories permettant, pour parler comme Paul Veyne, de dé-singulariser les faits étudiés et d'allonger la liste des questions qu'il saura poser à ses matériaux empiriques.

On comprend que la fiscalité provoque des révoltes, on comprend aussi que les contribuables s'y résignent: il faudrait savoir les circonstances dans lesquelles les uns se révoltent et les autres se résignent ${ }^{36}$.

Pour d'autres, au contraire, le recours à des procédures d'abstraction et de généralisation fonde la recherche en sciences sociales comme une «entreprise scientifique», au sens for ${ }^{37}$. Dans ce cas, il ne s'agit pas simplement de présenter la conceptualisation comme une manière de

34. Cf. les remarques d'Aline Rousselle (1987) sur le travail de problématisation de Michel Foucault.

35. Paul Veyne (1996: 57).

36. Raymond Aron (1971: 1325).

37. Cette idée d'une « science conceptuellement constituée » était centrale pour le projet durkheimien. Lors de la polémique de 1903, Simiand insistait ainsi sur la nécessité de «substituer à une pratique empirique, mal raisonnée, une méthode réfléchie et vraiment critique ». 
poser des questions différentes, mais bien de proposer des « modèles " et de construire des objets abstraits. Or, ces deux visions posent chacune des difficultés spécifiques.

Dans le premier cas, l'utilisation des termes « théories 》 ou " concepts » semble purement métaphorique. Si le chercheur se limite à mettre en forme et à raconter des « histoires », son seul objectif est alors de « faire comprendre ». On retombe donc sur une variante, plus ou moins subtile, de l'empirisme, dont les interprétations seront toujours approximatives ou flottantes. C'est du moins ce que semblait indiquer Paul Veyne (1969: 793 ; je souligne) :

Comme le romancier et l'historien, le sociologue, à ce jour, ne fait pas moins bien que le savant, il fait autre chose; il s'ensuit que son œuvre doit être utilisée autrement que celle d'un savant: elle ne nous révèle pas de vérités universelles, elle ne nous apporte pas de méthode, mais des suggestions et un exemple. L'Essai sur le don n'est pas une théorie du don, n'en construit pas de modèle, n'en donne pas la «formule » comme la chimie donne celle de l'oxyde carbonique, et l'économie, celle de l'utilité finale: il fait « comprendre» certaines conduites, au sens que ce mot a dans la vie de tous les jours, et il permet d'imaginer, par ressemblance ou par contraste, comment il faut comprendre d'autres conduites qui sont plus ou moins semblables à celles-là. Il dépouille les conduites de don de leur étrangeté exotique, il montre leur diversité et suggère par là une sorte de combinatoire de ces conduites.

Dans le deuxième cas, on peut au contraire pointer divers dangers de sur-théorisation ou de rigorisme scientiste. Le premier problème est ici lié au risque de négation de l'une des spécificités principales de toutes les sciences sociales et historiques : la commune historicité de leur objet d'étude ${ }^{38}$. J.-C. Passeron (2006) a ainsi beaucoup insisté sur le fait que cette caractéristique empêche les sciences sociales d'atteindre une généralité à valeur universelle (et anéantit, du même coup, toute prétention nomologique). Selon lui, les énoncés théoriques sur lesquels elles

38. Si les objets des sciences naturelles ont eux aussi une historicité, on remarquera avec G. Lenclud (1993 : 1226) que ce n'est peut-être pas tout à fait la même: «La manière qu'a une société d'être dans l'histoire est tout de même plus individualisée et changeante que celle d'un fleuve. Les hommes connaissent, espèrent, attendent, refusent ou désirent plus que le cours de l'eau qui n'a pas tout à fait choisi d'être ici dormant et là tumultueux ». 
s'appuient ne peuvent jamais s'autonomiser complètement du contexte singulier des faits étudiés (leur « ici et maintenant»). Or, il est évident que les chercheurs courent toujours le risque, dans leurs opérations de théorisation, de considérer leurs concepts, au moins à un niveau implicite, comme auto-suffisants, universels et quasi intemporels. Le recours à la théorie perd alors ce qui fait son intérêt - permettre une plus grande intelligibilité des faits observés - pour s'autonomiser et devenir le but même de la connaissance (c'est-à-dire une fin plutôt qu'un moyen) ${ }^{39}$. Et, au bout du compte, cette sur-théorisation conduit le chercheur à produire ce que Fernand Braudel (1958: 747) appelait « des vérités qui sont un peu trop celles de l'homme éternel ». S'appliquant à des situations tellement différentes ou tellement générales, l'usage « autonomisé » des concepts peut alors provoquer un aplatissement de la dimension historique et une déréalisation des processus étudiés (en particulier parce qu'il conduit à figer en des concepts uniformes des réalités et des expériences hétérogènes entre elles) $)^{40}$.

Par ailleurs, ce ne sont pas seulement les objets qu'étudient les chercheurs en sciences sociales qui sont pris dans une historicité spécifique, mais également les outils théoriques auxquelles ils ont recours. Ainsi, les concepts que nous utilisons ne relèvent pas d'un méta-langage transhistorique, propre à la seule vérité scientifique. Énoncés dans les termes de ce que Passeron appelle la « langue naturelle», les mots des sciences sociales portent en eux l'empreinte d'une époque et d'un lieu particuliers et, dans de nombreux cas, ils tendent à « vieillir » très vite (en particulier parce que leur contenu se modifie nécessairement avec le temps). Ainsi, les formes de conceptualisations sociologiques les plus en vogue durant de nombreuses décennies (« ajustement social », « appareil », « personnalité », « déviation », etc.) ont aujourd'hui grandement perdu de leur force d'attraction. Il en va de même en anthropologie ou en histoire, où de nombreux termes à certains moments incontournables (« culture de la pauvreté », « sous-culture », « mentalité ») ont pratiquement disparu du

39. Comme l'a écrit Passeron (2003 : 118) en référence au « théorétisme (marxiste, adornien, gurvitchien, marcusien, herméneute, ontologiste, peu importe) », dans ce cas, « le travail sur les concepts tient lieu de terrain ». Diverses métaphores permettent de mettre en garde les chercheurs trop portés à la théorisation. On reprochait ainsi à Erik Olin Wright de charger son sac à dos de concepts pour un voyage qu'il n'a jamais fait. On se rappellera également la métaphore du Métier de sociologue à propos du malade « qui passait son temps à essuyer ses lunettes sans jamais les chausser ».

40. Cf. « Tentons l’expérience », Annales. ESC, n6, 1989, p. 1319. 
langage des chercheurs. Loin de faire un usage mécanique des concepts qu'il mobilise dans ses analyses, le chercheur se doit donc de veiller à toujours les problématiser et les historiciser. Il est pourtant très facile de glisser vers des usages contestables de la théorisation.

Une première difficulté vient de ce que l'on considère parfois le rapport théorie/empirie comme une relation d'illustration ou de remplissage. Dans ce cas, la théorie et les concepts précèdent l'enquête empirique et les matériaux viennent s'y adapter ${ }^{41}$. C'est l'un des reproches, au-delà des limites empiriques de son travail déjà mentionnées, qui a été fait à Michel Foucault dans son usage des archives. Dans bien des cas, il semblait en effet ne faire référence aux documents que pour illustrer ou justifier une théorie ou une idée déjà présente (plutôt que de s'immerger dans les archives pour y apprendre quelque chose de nouveau). Alain Cottereau (1996) était ainsi très critique du chapitre sur « les corps dociles » de Surveiller et punir, dans lequel Foucault s'appuyait sur la lecture commentée d'un règlement d'atelier parisien de 1809. Pour Cottereau, Foucault avait sélectionné et mis hors contexte les seuls articles du règlement qui venaient à l'appui de sa thèse sur les « technologies disciplinaires », tandis que tous ceux qui ne « collaient » pas (au sens où ils invalidaient sa conception du pouvoir) étaient passés sous silence. Au final, les concepts peuvent donc donner l'illusion de la compréhension, mais ne servir en réalité qu'à confirmer ce que l'auteur savait déjà avant de faire l'enquête ${ }^{42}$.

Une deuxième difficulté vient de ce que l'on pourrait appeler les usages mécaniques ou routinisés d'une théorie. C'est le reproche qui a souvent été fait à certains disciples de Pierre Bourdieu, que l'on a accusés de reprendre, sans véritablement l'interroger, l'appareil concep-

\footnotetext{
41. L'objectif du chercheur peut être de confirmer cette théorie ou au contraire de la contester. Dans les deux cas, c'est pourtant bien la théorie comme telle (et non l'univers étudié) qui oriente la réflexion $\mathrm{du}$ chercheur. Le risque est alors de faire un usage purement rhétorique de «l'exemplification». On peut alors parler d'une " pyramide inversée ", puisque au lieu de partir d'une large base empirique qui servirait d'assise à une réflexion conceptuelle, le fondement véritable du travail du chercheur semble être la « pointe » conceptuelle, qui soutient la large collection d'éléments empiriques mobilisés a posteriori. Cf. Stoczkowski, 2001. De façon similaire, J. Derrida (1967 : 184) parlait, à propos de Tristes tropiques, de « la pointe de l'incident [qui] supporte un énorme édifice théorique ».

42. Le problème n'est pas très différent dans le cas des enquêtes de terrain ou des statistiques. On rappellera à ce sujet la critique sévère adressée par Claude Grignon (2002) à Christian Baudelot, qui se voyait accusé de vouloir « enrôler les statistiques au service d'une cause et [de] passer en revue des faits qui n'avaient plus qu'à s'aligner sur une thèse, comme à la parade ».
} 
tuel qu'il avait développé (et notamment les trois notions centrales de champ, habitus et capital). J.-C. Passeron (2003) parlait à ce sujet de la « reproduction de l'idiome » comme d'une « imitation stérile chez ceux qui ne peuvent s'empêcher de promouvoir leur maître de recherche en maître à penser ». Au lieu de provoquer un « engagement » du chercheur avec ses matériaux, les concepts contribuent dans ce cas à en faire un traitement « pré-formaté $»^{43}$. De façon similaire, on a reproché à certains chercheurs se servant du concept de "genre» de postuler l'existence « d'effets de genre » à l'intérieur du monde étudié, plutôt que d'étudier au cas par cas le fonctionnement pratique et concret de ces effets ${ }^{44}$. On tombe alors dans ce que Revel et Lepetit appelaient une « tautologie des descriptions à l'intérieur de catégories prédéterminées ou de notions globales abstraites $»^{45}$. Poussé à l'extrême, ce recours non problématisé aux concepts conduit à des usages que l'on pourrait qualifier de purement cosmétiques ou rhétoriques. C'est-à-dire que le chercheur se contente de " plaquer» sur le réel étudié des postulats reçus ou des formules toutes faites, dont le succès est bien souvent proportionnel au flou qui entoure leur signification. Une fois que l'on nous a dit, par exemple, que la migration est un «phénomène social total » ou que le voyage en avion est un « rite de passage ", il n'est pas sûr que nous ayons appris grand chose de particulièrement nouveau ni sur la migration ni sur les voyages en avion (cf. Stoczkowski, 2001).

Par ailleurs, certains chercheurs ont tendance à oublier que le caractère partial et partiel des recherches en sciences sociales ne se joue pas seulement au moment de la collecte des matériaux (comme nous l'avons déjà signalé), mais également lors de la théorisation. Les concepts ont en effet un double pouvoir de révélation et de dissimulation. L'introduction d'une catégorie nouvelle permet bien souvent de mettre en évidence certains mécanismes restés jusque-là invisibles ou impensables. Mais elle implique inévitablement une lecture orientée des matériaux et un point de vue qui reste fragmentaire. On conviendra par exemple que la notion d'habitus,

43. Certains parlent de «moissonneuses-batteuses théoriques » ou de «sociologie bulldozer » pour désigner les approches qui - ne prêchant le plus souvent que les convertis - tendent à enfermer les réalités sociales dans des schémas explicatifs établis d'avance.

44. Il ne s'agit pas évidemment pas ici de minimiser l'intérêt de ce concept. C'est précisément parce que son pouvoir heuristique est évident qu'il me permet de pointer des difficultés inhérentes à l'ensemble des concepts mobilisés par les sciences sociales.

45. « Tentons l'expérience », Annales. ESC, 1989. 
que Bourdieu a fortement contribué à institutionnaliser au sein des sciences sociales, a joué un rôle important pour dénoncer un certain nombre de fausses alternatives encombrant le travail des chercheurs (les oppositions entre l'individuel et le social, entre la liberté et le déterminisme, le subjectif et l'objectif, etc.). Pourtant, s'il avait un pouvoir évocateur indéniable, ce concept avait aussi, comme n'importe quel autre, des limites, fonctionnant, à de nombreux niveaux, comme une «boîte noire $»^{46}$. De même, la mise au jour de quatre types de violence par Bourgois, à laquelle nous avons fait référence, a indéniablement permis de rendre visibles ou pensables des faits qui ne l'étaient pas, mais en aucun cas elle ne représente une typologie définitive ou exhaustive (c'est-à-dire qui permettrait de décrire parfaitement et de classer de manière réaliste des registres d'expérience ${ }^{47}$ ). Il sera donc toujours possible de recourir à de nouvelles grilles catégorielles qui organiseront de nouvelles descriptions et produiront des conclusions différentes. Quelle que soit sa richesse, une orientation théorique ne permettra jamais d'épuiser la complexité du fonctionnement du monde social (ou d'en proposer une explication « en dernière analyse »). En ce sens, sa fonction n'est pas d'aider à déchiffrer une intelligibilité immanente, mais d'appuyer un travail de «construction» des faits, qui implique inévitablement de choisir entre des systèmes d'interprétation multiples et concurrents ${ }^{48}$.

Au final, on voit bien les multiples fragilités inhérentes à la théorisation. Les concepts sont certes des abstractions indispensables pour une meilleure compréhension de phénomènes a priori peu intelligibles. Et pourtant cette compréhension passe inévitablement par une certaine simplification ou reformulation des « faits », au sens où, nous l'avons vu, l'introduction de certains concepts favorise nécessairement certaines interprétations au détriment des autres (certaines manières de " découper » le réel, pour reprendre la métaphore du boucher de Phèdre $)^{49}$. C'est ce qui explique que le caractère opératoire des concepts est toujours provisoire ou relatif:

46. Sur l'idée de l'habitus comme « boîte noire », cf. par exemple les travaux de L. Wacquant (2010) ou de B. Lahire (1998). Encore une fois, il ne s'agit pas ici d'attaquer telle ou telle école mais de rendre compte des mécanismes communs aux chercheurs en sciences sociales.

47. On conviendra, pour reprendre l'expression de Bergson (citée par Bloch), qu'il serait illusoire de penser que les classifications puissent coller aux « lignes mêmes du réel ».

48. Le paradoxe de cette pluralité de théories concurrentes est qu'une nouvelle proposition peut être reconnue comme légitime sans pour autant remettre en cause la validité d'autres propositions dont on pourrait pourtant penser qu'elles sont contradictoires. Cf. J.-C. Passeron (1994).

49. Yvon Thébert (1978) montrait par exemple, que le simple fait d'utiliser le concept de « romanisation » pour décrire l'Afrique antique supposait toujours de mettre l'accent sur « une politique de romanisation 
ils ne valent que tant que l'on considère qu'ils ajoutent quelque chose à l'analyse. Mais, tôt ou tard, on pourra toujours leur reprocher d'être inadéquats aux réalités qu'ils représentent ou de fausser les perspectives. Gérard Noiriel (1986) expliquait ainsi qu'il avait été amené à abandonner progressivement certaines notions familières pour les historiens - celle de 《 tradition » ou de « mentalité collective »-pour leur préférer le concept de « reproduction sociale ». Ce dernier avait l'avantage de montrer, par rapport aux autres notions, que la « permanence » ou la « durée » d'une société n'avaient rien de spontané, mais qu'elles étaient le produit d'un travail social de renouvellement. Pourtant, comme le reconnaissait Noiriel, ce même concept ne constituait pas une «nouvelle panacée », mais simplement un outil utile dans un dispositif de recherche (qui a d'ailleurs été lui-même partiellement critiqué depuis).

Et ces reproches peuvent prendre des directions contradictoires, puisque les concepts, en tant qu'ils sont ce que Passeron appelle des « semi-noms propres », doivent remplir des exigences antagoniques. Ils doivent permettre tout à la fois, de respecter la singularité des faits étudiés (puisque, considérés dans tous leurs détails, ces derniers ne se confondent avec aucun autre et qu'ils ne se reproduiront pas). Et, dans le même temps, de les "dé-singulariser», en soulignant ce qu'ils ont en commun avec d'autres d'une même catégorie. Dans certains cas, on pourra donc reprocher aux concepts de « trahir » le réel du fait de leur trop grande globalité (conduisant par exemple à des rapprochements infondés ou à l'homogénéisation de faits dont l'interprétation reste incertaine). On s'est ainsi interrogé, dans les années 1970, sur la tentation qu'avaient les historiens de l'Afrique d'emprunter aux médiévistes la notion de « féodalité » : plutôt que suggérer des comparaisons légitimes et fécondes entre l'Europe médiévale et l'Afrique pré-coloniale, E. Terray (1973) laissait entendre qu'elle produisait au contraire des contresens. Dans d'autres cas, on insistera au contraire sur leur caractère trop restreint et sur leur incapacité à s'élever au dessus du vécu et de l'expérience (un élargissement de la notion permettant de rapprocher des registres d'expériences jusque là pensés séparément). L'important n'est donc pas d'arriver à des formes de théorisation justes et définitives, mais d'adopter une attitude inquiète et réflexive. Si l'on ne saurait éviter totalement de projeter

de la part de Rome ». Ceci alors même que la majorité des chercheurs admettaient ouvertement ou implicitement l'existence de multiples nuances dans ses modalités d'application. 
dans l'objet étudié nos catégories d'observateur (puisque, nous l'avons vu, les catégories du chercheur sont toujours des catégories situées et construites), nous pouvons par contre être conscients que les modes de groupement et d'articulation des faits constituent toujours des procédés heuristiques. Enjeux de débats et de choix, ils nous servent d'appui pour construire nos interprétations, mais ne peuvent jamais donner accès à la réalité permanente de la nature ${ }^{50}$.

\section{Conclusion: Peut-on fixer une vraie corde à un crochet peint au mur $?^{51}$}

Les sciences sociales se considèrent généralement comme des " sciences empiriques de l'interprétation ${ }^{52}$ », c'est-à-dire comme des sciences qui impliquent à la fois la constitution de données empiriques, puis la mise en œuvre de procédures analytiques permettant l'interprétation de ces données. Et pourtant, nous l'avons vu, ces deux dimensions sont également problématiques. Premièrement, ces sciences visent la réalité des processus sociaux tout en affirmant qu'il est impossible d'accéder au « réel », que les faits n'existent pas indépendamment de celui qui les constate. Deuxièmement, ces sciences affirment l'importance de la théorie et de la conceptualisation pour l'interprétation des matériaux, mais elles reconnaissent dans le même temps leur incapacité à formaliser, à « démontrer », ou à s'élever au général ou aux lois. Enfin, la volonté de réunir ces deux dimensions, dans un ensemble composite où le théorique vient se mêler à l'expérience vécue, complique encore la situation. L'articulation entre les deux ne va jamais de soi et, comme l'a remarqué Paul Veyne, rien n'est plus instable que les mixtes. Pourtant, c'est bien là une condition qu'il semble falloir assumer, et qui paraît une caracté-

50. C'était bien, me semble-t-il, l'objectif de la notion d'idéaltype que proposait Max Weber de manière à faire le lien entre singularités historiques et abstractions généralisantes. On remarquera que Weber avait parfaitement explicité le caractère inextricable de la situation des chercheurs : « Plus le type idéal est net et univoque, plus il est en ce sens étranger à l'univers concret, et plus il rend de services à la terminologie, à la classification et à l'heuristique ». Dans une autre tradition, Durkheim et Mauss conseillaient eux aussi de ne jamais accepter comme évidents les « cadres de classement » utilisés pour l'analyse: ces derniers devaient faire l'objet d'un effort permanent de discussion, de remaniement ou de réadaptation. 51. D’après Krzysztof Pomian cité par Ruben Cesar Fernandes, «Vision du monde et compréhension historique : sur le Rousseau de Baczko », Annales. ESC, n², 1971, p. 395.

52. Passeron (2006: 64). 
ristique indépassable du statut épistémologique des sciences sociales. Celles-ci ne peuvent certes pas prétendre à une connaissance objective du monde social (qui passerait par l'établissement de causes, de lois ou de modèles, au sens strict), mais elles n'ont pas non plus vocation à devenir un genre littéraire narratif, s'en tenant à l'étude de cas singuliers. Le chercheur est à la fois contraint par la réalité empirique à laquelle il s'intéresse (les hommes ou les événements sont ce qu'ils sont et ni le chercheur ni personne n'y changera rien) mais également relativement « libre » dans ses questions ou dans sa construction (il se taille son propre champ d'enquête). S'il ne reproduira jamais l'expérience concrète des personnes, il doit pourtant s'appuyer sur elle pour construire des objets plus ou moins éloignés des données empiriques. Au final, il nous semble donc nécessaire de prendre au sérieux et d'assumer cette contradiction comme indépassable plutôt que de se dérober devant les apories de la situation. Les problèmes mentionnés n'ont pas trouvé de solution définitive et il serait dangereux de faire croire le contraire. D'une certaine façon, le projet des sciences sociales avance toujours sur le fil du rasoir et il ressemble à celui de vouloir faire des carrés ronds.

Pourtant, l'explicitation et l'acceptation de cette double contradiction ne conduit pas nécessairement à un constat d'échec. Elle permet en particulier de remettre en cause certaines «mauvaises querelles » qui, dans les sciences sociales, ont la vie dure. L'une d'entre elles est liée à l'opposition objectivité/subjectivité ${ }^{53}$. Trop souvent, cette alternative continue à structurer, au moins implicitement, nos perceptions et « l'objectivité » reste, sinon l'idéal, du moins l'horizon implicite d'une quantité de chercheurs ${ }^{54}$. Or, si l'on commence par reconnaître que les sciences sociales et historiques ne peuvent ni atteindre la «réalité » entière et définitive, ni dégager des « lois », on ne peut plus les juger suivant ce critère. Comment leur reprocher en effet de ne pas atteindre l'objectivité proprement dite si cela ne fait clairement pas partie de leur horizon de possibilité? Mais, l'on ne peut pas s'arrêter là, sous peine de retomber dans la position symétrique, qui consiste à penser que, dans ce cas, les sciences sociales sont nécessairement « subjectives », et que l'on peut donc les assimiler à une forme de littérature. Malgré tout ce qui les sépare, ces deux positions ont en commun d'accepter l'opposition objectif/

53. Et ses variantes autour de la synonymie entre objectivité, réfutabilité et scientificité.

54. Cf. Busino, 2003. 
subjectif comme légitime. Or, il me semble que l'explicitation de cette double tension nous invite à faire un pas de côté. L'opposition pertinente n'est pas celle-ci mais celle qui oppose sciences mécaniques et sciences réflexives. En ce sens, le fait de renoncer à l'idéal d'une connaissance objective de la réalité sociale, au sens habituel de l'expression, n'implique pas d'abandonner l'horizon général qui est, depuis longtemps, celui de ces sciences sociales (dont Max Weber disait déjà qu'elles étaient condamnées à rester " éternellement jeunes »): se donner les moyens de mieux comprendre les mécanismes du monde social. Cela n'implique pas non plus de renoncer à tout idéal de méthode ou de nous laisser guider par des présupposés inconscients ou par les hasards de notre recherche. Au contraire, la pratique réflexive ("imaginative », aurait dit Mills) des sciences sociales est porteuse d'exigences supplémentaires quant aux modalités d'élaboration des connaissances. Elle oblige le chercheur à produire un savoir qui ne serait pas aveugle aux conditions de son élaboration et à revendiquer la nécessité d'un re-travail permanent des instruments et cadres de la recherche, ainsi que des catégories mises en œuvre. Au final, il nous faut donc bien accepter que les chercheurs ont nécessairement à faire des choix, qu'ils ne peuvent entrer dans tous les détails ou qu'ils sont contraints d'ignorer des thèmes ou des niveaux. Le problème n'est pas là. Ce qui est problématique, c'est de faire ces choix sans en avoir conscience, ou sans le dire.

\section{Bibliographie}

« Tentons l’expérience », 1989. Annales. ESC, n6, p. 1317-1323.

«Présentation », 1997. Annales. HSS, n5, p. 963-967.

Aron Raymond, 1938. Introduction à la philosophie de l'histoire : essai sur les limites de l'objectivité historique (Paris, Gallimard).

-, 1971. «Comment l'historien écrit l'épistémologie: à propos du

livre de Paul Veyne », Annales. ESC, nº, p. 1319-1354.

Bцoch Marc, 1952 [1949], Apologie pour l'histoire ou métier d'historien,

(Paris, Librairie Armand Colin).

Bourdieu Pierre, ed., 1993. La Misère du monde (Paris, Éditions du Seuil). Bourdieu Pierre, Luc Boltanski, Robert Castel \& Jean-Claude Chamboredon, 1965. Un Art moyen, essai sur les usages sociaux de la photographie (Paris, Éditions du Minuit). 
BouRgols Philippe, 2002. « La violence en temps de guerre et en temps de paix. Leçons de l'après-guerre froide: l'exemple du Salvador. Partie I », Cultures \& Conflits, 47, p. 81-116.

Braudel Fernand, 1958. "Histoire et Sciences sociales: La longue durée », Annales ESC, n ${ }^{\circ}$, p. 725-753.

Busino Giovanni, 2003. "Sciences sociales et histoire", Revue européenne des sciences sociales, XLI-127, p. 119-171.

Clifford James and George Marcus (eds.), 1986. Writing Culture: the Poetics and Politics of Ethnography (Berkeley: University of California Press).

CoHn, Bernard S, 1962. "An Anthropologist among the Historians". South Atlantic Quarterly, n61, p. 13-28.

CotTereau Alain, 1996. « La gestion du travail, entre utilitarisme heureux et éthique malheureuse », Le Mouvement social, n¹75, p. 7-29.

DERrIDA Jacques, 1967. De la grammatologie (Paris, Minuit).

Evans-Pritchard E. E., 1950. "Social Anthropology: Past and Present the Marett Lecture, 1950”, Man, n50, p. 118-124.

Febvre Lucien, 1943. " Propos d'initiation : vivre l'histoire », Mélanges d'histoire sociale, $\mathrm{n}^{\circ} 3$, p. 5-18.

-, 1953a. «De Mitra-Varuna à Clio », [note critique], Annales. ESC, n'1, p. 67-69.

-, 1953. «Pro parva nostra domo : scolies sur deux articles belges », Annales. ESC, n², p. 512-518.

-, 1956. «De la création en histoire. À la mémoire de Georges Jamati », Annales. ESC, n³, p. 292-295.

FrIEDMANN Georges, 1960. "Société et connaissance sociologique ", Annales. ESC, n¹, p. 9-17.

Griaule Marcel, 1957. Méthode de l'Ethnographie (Paris, Presses Universitaires de France).

Grignon Claude, 2002. « Comment peut-on être sociologue? », Revue européenne des sciences sociales, XL-123, p. 181-225.

HARTOG François, 1978. "Paul Veyne naturaliste: l'histoire est un herbier ", Annales. ESC, vol. 33, n², p. 326-330. (Sur le livre de Paul Veyne, L'inventaire des différences, Paris, Seuil, 1976).

LAHIRE Bernard, 1996. «Risquer l'interprétation. Pertinences interprétatives et surinterprétations en sciences sociales ", Enquête. Anthropologie, Histoire, Sociologie, Interpréter, surinterpréter, n³, p. 61-87. 
LAHIRE Bernard, 1998. L'Homme pluriel : les ressorts de l'action (Paris, Nathan).

LENCLUD Gérard, 1991. «Le nomologique et le néant », Études rurales, n¹21-122-123-124, p. 255-263.

-, 1993. « La statue du commandeur (note critique) ». Annales. ESC, n 5 , p. 1221-1230.

LÉvi-Strauss Claude, 1960. «L'anthropologie devant l'histoire », Annales. ESC, ${ }^{\circ} 4$, p. 625-637.

LÉvy René and Philippe RoBert, 1984. « Le sociologue et l'histoire pénale », Annales. ESC, n², p. 400-422.

Malinowski, Bronislaw. 1986 [1922]. «Introducción: Objeto, método y finalidad de esta investigación »: Los Argonautas del Pacífico Occidental (Barcelona, Península).

Mandrou Robert, " Géographie humaine et Histoire sociale », Annales, ESC, n4, 1957. p. 619-627.

Marrou Henri-Irénée, 1954. De la Connaissance historique, Paris, Seuil. NAEPELS Michel, 2006. «Quatre questions sur la violence », L'Homme, $\mathrm{n}^{\circ} 177-178, \mathrm{p} .487-495$.

NoIRIEL Gérard, 1986. «L'immigration en France, une histoire en friche », Annales. ESC, n ${ }^{\circ}$. p. 751-769.

,- 1989 . « Pour une approche subjectiviste du social », Annales. ESC, $\mathrm{n}^{\circ} 6$, p. 1435-1459.

Passeron Jean-Claude, 2006 [1991]. Le Raisonnement sociologique. L'espace non-poppérien du raisonnement naturel (Paris, Éditions Nathan).

-, 1994. «De la pluralité théorique en sociologie: théorie de la connaissance sociologique et théories sociologique », Revue européenne des sciences sociales, $\mathrm{n}^{\circ} 99, \mathrm{p} .71-116$.

-, 2003. "Mort d'un ami, disparition d'un penseur », Revue européenne des sciences sociales, XLI-125.

Peter Jean-Pierre, 1964. « L'histoire à l'épreuve de la guerre d'Espagne », Annales ESC, ${ }^{\circ} 1$, p. 142-174. [sur Hugh Thomas, La guerre d'Espagne, Paris, R. Laffont, 1961].

Roubaud Jacques, Le grand incendie de Londres, Assertion \#11.

Rousselle Aline, 1987. "Michel Foucault, Histoire de la sexualité, t. II : L'usage des plaisirs, t. III: Le souci de soi», Annales. ESC, $\mathrm{n}^{\circ} 2$, p. 317-321. 
DE SARDAN Jean-Pierre, 1993. « L'espace wébérien des sciences sociales ». Genèses, 10, p. 146-160.

Schnapp Alain, Pauline Schmitt and Jean Andreau, 1978. « Paul Veyne et l'évergétisme ", Annales. ESC, n², p. 307-325 [sur Paul Veyne, Le pain et le cirque, sociologie religieuse d'un pluralisme politique, Paris, Seuil, 1976].

Schwartz Olivier, 1993. "L'empirisme irréductible », dans ANDERSON

Niels, Le Hobo : sociologie du sans-abri (Paris, Nathan).

Simiand François. " Méthode historique et science sociale. ( ${ }^{\mathrm{e}}$ partie) 》, 1903. Revue de synthèse historique, p. 129-157.

Sperber Dan, 1996. La contagion des idées (Paris, Odile Jacob).

Stengers Jean, 1953. «Marc Bloch et l'histoire ", Annales. ESC, n³, p. 329-337.

THÉBERT Yvon, 1978. «Romanisation et déromanisation en Afrique: histoire décolonisée ou histoire inversée? ", Annales. ESC, ${ }^{\circ} 1$, p. 4-82.

Stoczkowski Wiktor, 2001. «Rire d'ethnologues », L'Homme, n¹60, p. 91-114.

Terray Emmanuel, 1973. «État, tradition, technologie en Afrique: note critique », Annales. ESC, n5, p. 1331-1338 [sur Jack Goody, Technology, tradition and the state in Africa, Londres, Oxford University Press, 1971].

Testart Alain, 1991. Pour les sciences sociales : Essai d'épistémologie (Paris, Christian Bourgois).

Veyne Paul, 1969. «Panem et circenses : l'évergétisme devant les sciences humaines ", Annales. ESC, n³, p. 785-825.

-, 1996 [1971]. Comment on écrit l'histoire, Paris, Seuil.

-, 1972. «Delio Cantimori » [recension], Annales. ESC, n³, p. 668-669.

WACQUANT Loïc, 2010. «L'habitus comme objet et méthode d'investigation. Retour sur la fabrique du boxeur », Actes de la recherche en sciences sociales, $\mathrm{n}^{\circ} 184$, p. 108-121.

White Hayden, 1973. Metahistory: The Historical Imagination in Nineteenth-Century Europe (Baltimore, The Johns Hopkins University Press). 